\title{
Frequency-angular spectra of the stimulated Raman scattering parametric components under self-action
}

\author{
A.I. Ivanisik ${ }^{1}$, O.Iu. Isaenko and P.A. Korotkov \\ Taras Shevchenko Kyiv National University, \\ 4g, Academician Glushkov Avenue, 03127 Kyiv, Ukraine \\ ${ }^{I}$ Phone: +38 (044)-526-0570; e-mail: aivan@univ.kiev.ua
}

\begin{abstract}
It's theoretically proved that in self-focusing mode of exciting radiation, selfphase modulation, and the relaxation oscillations of the nonlinear polarization amplitude, the frequency-angular structure of the parametric components of the stimulated Raman scattering has special features. Maximum of radiation energy shifts relative to Raman frequencies, and this shift depends on the scattering angle. The largest Stokes shift (about $-10 \mathrm{~cm}^{-1}$ ) of the maximum energy in the spectrum of parametric components corresponds to axial radiation and is determined by the size of the focal area, time of polarization existence as well as mismatch of the polarization wave vectors and parametric component. The obtained estimations of the self-phase modulation influence on radiation in the case of relaxation oscillations show that this influence is insignificant. The effect has been interpreted in terms of short-term phase matching.
\end{abstract}

Keywords: stimulated Raman scattering, self-focusing, self-phase modulation, relaxation oscillations.

Manuscript received 27.08.12; revised version received 25.09.12; accepted for publication 17.10.12; published online 12.12.12.

\section{Introduction}

Stimulated Raman scattering (SRS) is widely used to control the shape of optical pulses and their spectra, the statistics of laser radiation and its spatial distribution, to create devices conjugating the wave front [1], and to amplify weak signals in optical fibers [2]. On the other hand, the parameters of the scattered radiation, especially spectrum, spatial-temporal structure, and coherence are also an important source of information about the nature of the scattering process itself and properties of the scattering medium.

However, many features of SRS remain unclear. In particular, in self-focusing liquids, even in the nanosecond duration range of excitation laser pulse, the spectral lines broadening of SRS parametric components significantly exceeds the line width of spontaneous Raman scattering. The proposed list of mechanisms, classification of such broadenings [3-6] is not conclusive and needs further details and additions. In particular, this paper examines the action of self-phase modulation and relaxation oscillations of the nonlinear polarization amplitude under self-focusing conditions.

\section{Theoretical consideration}

In our studies, we assumed that the experimental threshold of SRS in self-focusing liquids is mainly determined by the threshold of exciting radiation selffocusing. If self-focusing threshold is exceeded 2-3 times, then, in the case of light pulse duration close to $10 \mathrm{~ns}$ and cell length close to $10 \mathrm{~cm}$, the focal area appears first at the output side of the medium. The growth of instantaneous power at the front of the laser pulse is accompanied by the movement of the focal area in the direction of input side of the medium, i.e. towards the laser source. When the power reaches its maximum value, the focus stops at the minimum distance from the input border ("turning point"), and at the drop of the laser pulse the focus moves in the opposite direction, to the output side [7]. The pass from the input border of the medium to the center of focal area is considered as the "input side" of the medium, while pass from the center of focal area to the output border is taken into account as the "output side".

The amplitude of induced nonlinear polarization, which is the source of parametric components, reaches 
the maximum value at the focal area [8]. Due to the focal area movement the maximum polarization amplitude area moves as well. As shown in [6], this itself causes the frequency shift (a few $\mathrm{cm}^{-1}$ ) of parametric components against the Raman frequencies. The shift occurs in the direction of the frequency, which satisfies the condition of amplitude-phase matching: the condition of synchronous movement of focal area and the area of polarization phase synchronism and the generated wave.

However, most of the time at the top of the laser pulse power, when the focal area is near the turning point, it has a velocity close to zero. In this case, the frequency shift under the mentioned mechanism [6] is insignificant as compared to the frequency shift under other mechanisms. In our opinion, the shift can be caused by the mechanism associated with the relaxation oscillations of the nonlinear polarization amplitude.

Under relaxation oscillations, the SRS mode in the focal area is developed not only in exciting laser beam direction but also in the opposite direction. If the focal area is moving slowly or is stationary (as at the turning point), then, in addition to the Stokes wave of the SRS in the opposite direction, the Stokes wave of the stimulated Mandelstam-Brillouin scattering can be generated, which requires a longer developing time than SRS. The radiation scattered in the opposite direction meets the exciting laser beam and amplifies itself, contributing to the effective exhaustion of exciting radiation. As a result of exciting radiation exhaustion, the self-focusing disappears, and its disappearance stops the scattering. Over the time, during which the earlier scattered radiation leaves the medium, the threshold of selffocusing is reached again and the process repeats.

Oscillation period $T$ of the Stokes component intensity variance is determined by the time needed for the light to pass the distance from the input side of the medium to the focal area and backward [9]: $T \approx 2 z_{f} n / c$, where $z_{f}$ is the self-focusing distance; $c$ - speed of light in vacuum; $n$ - refractive index of light in the medium. For $z_{f}=10 \mathrm{~cm}$ and $n=1.5$, the oscillation period is $T \approx 1 \mathrm{~ns}$.

The amplitude of nonlinear polarization induced in the focal area, which is the source of SRS parametric components, oscillates simultaneously with the Stokes components intensity at the period of $T$. In each oscillation the increase of the polarization amplitude occurs during the SRS development time $\tau_{g}$, which is $\sim 1$ ps [7]. The decline in the amplitude of polarization occurs during the time $\tau_{d}$ of light passing through the focal area, but is not less than the duration of the longitudinal relaxation of Raman-active transition, for the focal area length $b=0.1 \mathrm{~cm}$ and $n=1.5$, $\tau_{d} \approx b n / c=5 \mathrm{ps}$. Thus, we can expect that the time $\tau$ of polarization existence in each of the oscillations lies within $\tau=1 \ldots 5$ ps by the order of magnitude for typical
Raman-active self-focusing liquids, and it is much smaller than the oscillations period $T$. This allows limiting the review to the calculation of frequencyangular structure of the parametric SRS components spectra for one sub-pulse of exciting self-focused radiation. Oscillation periodicity leads only to more substructures in the spectra in scale of $1 / T<1 / \tau$, which is difficult to observe experimentally, as $1 /(2 \pi c T) \sim 0.01 \mathrm{~cm}^{-1}$.

\section{Calculation methodology}

To simplify calculations, it's assumed that all waves are polarized identically and nonlinear polarization $P^{N L}$, which is the source of some parametric component in the focal area, can be represented as:

$P^{N L}(t, \mathbf{r})=P_{0}^{N L} F_{t}(t) F_{\perp}\left(r_{\perp}\right) F_{z}\left(z-z_{f}\right) \exp \left[-i \varphi_{p}(t, \mathbf{r})\right]$,

where $P_{0}^{N L}$ is the maximum polarization amplitude in the focal area; $F_{t}(t)$ is the function that describes the temporal change in the amplitude due to polarization relaxation oscillations; $F_{\perp}\left(r_{\perp}\right)$ is the lateral distribution function of the polarization amplitude, which value is determined by the distance $r_{\perp}$ from the axis $z$; $F_{z}\left(z-z_{f}\right)$ is the longitudinal distribution function of the polarization amplitude, which argument is the distance $\left(z-z_{f}\right)$ from the focal area center; $\varphi_{p}(t, \mathbf{r})$ is the phase of the polarization; $t$ is time, and $\mathbf{r}$ is the radius vector.

There is a connection between each Fourier component of the polarization

$P_{\omega}^{N L}=\int_{-\infty}^{\infty} P^{N L}(t, \mathbf{r}) \exp (i \omega t) d t$,

and generated field

$E_{\omega}=\int_{-\infty}^{\infty} E(t, \mathbf{r}) \exp (i \omega t) d t$

in the far field area. This connection in the case of small scattering angles $\theta$ can be represented as [6]:

$$
E_{\omega}(\omega, \mathbf{R})=\frac{\omega^{2}}{c^{2} R} \exp [i k R] \int_{V} P_{\omega}^{N L}(\omega, \mathbf{r}) \exp [i \mathbf{k r}] d^{3} r
$$

where $\mathbf{R}$ is the vector that connects any point within the area $V$ of polarization and an observation point; $\mathbf{k}=\mathbf{k}(\omega)=(\omega n / c) \mathbf{R} / R-$ wave vector of parametric component field with length $k$, unidirectional with $\mathbf{R}$. The formula for frequency-angular energy density can be obtained as:

$$
W_{\omega \theta}=\frac{\omega^{4} n}{8 \pi^{2} c^{3}}\left|\int_{V} d^{3} r \int_{-\infty}^{+\infty} d t P^{N L}(t, \mathbf{r}) \exp [i(\omega t-\mathbf{k r})]\right|^{2},
$$


which after substitution of $P^{N L}(t, \mathbf{r})$, specification of the parametric process and the functions $F_{t}(t), F_{\perp}\left(r_{\perp}\right)$, $F_{z}\left(z-z_{f}\right)$ in (1) allows to calculate the frequencyangular spectrum of the selected parametric component of SRS under self-focusing mode of exciting radiation and the relaxation oscillation of the nonlinear polarization amplitude.

\section{Calculation results}

To obtain the expression of energy density $W_{\omega \theta}$, it's necessary to determine the phase of nonlinear polarization, i.e. find the phase delay of radiation that includes delays at the input and output sides of the medium. For simplicity, let us consider only axial radiation, allowing to assume that $x=0, y=0$, resulting in $F_{\perp}\left(r_{\perp}\right)=1$.

The wave vector length $k$ at the focal area increases up to some value $\Gamma$. The maximum of this value $\Gamma=\Gamma_{f}$ is gained at $z=0$, where the center of the focal area is located $\left(z_{f}=0\right)$, and $t=0$, when light intensity in the focal area is maximal. In this case $\Gamma$ can be determined as:

$$
\Gamma(t, z)=\Gamma_{f} \exp \left[-(z / b)^{2}\right] \exp \left[-(t / \tau)^{2}\right] .
$$

A fragment of laser pulse, which is located in a point $z$ at the moment of time $t$, was located at some point $\xi$ (on the pass from input border to the point $z$ ) at $t^{\prime}=t-(z-\xi) / v_{g}$, where $v_{g}$ is the group velocity of laser radiation. According to the specified considerations phase of laser changes to:

$$
\delta \varphi_{L}(t, z)=\Gamma_{f} \int_{-L / 2}^{z} \exp \left[-(\xi / b)^{2}\right] \exp \left[-\left(\left(t-(z-\xi) / v_{g}\right) / \tau\right)^{2}\right] d \xi,
$$

where $L$ is the length of the medium with the center at $z=0$. The medium border in our model is located at a considerable distance from the focal area, i.e. $L / 2>>b$, which is equivalent to replacing the lower limit of integral (7) with $-\infty$. Since we are interested in the maximum amplitude of polarization, the phase $\delta \varphi_{L}$ should be determined for coordinate and time $z=0$, $t=0$ :

$$
\begin{aligned}
& \delta \varphi_{L}(0,0)=\Gamma_{f} \int_{-\infty}^{0} \exp \left[-(\xi / b)^{2}-\left(\xi /\left(v_{g} \tau\right)\right)^{2}\right] d \xi= \\
& =\frac{\sqrt{\pi}}{2} \Gamma_{f} b / \sqrt{1+\left(b /\left(\cup_{g} \tau\right)\right)^{2}} .
\end{aligned}
$$

The same initial conditions allow to calculate analytically the following derivatives of the phase:

$$
\begin{aligned}
& \frac{\delta \varphi_{L}}{\partial t}(0,0)=\Gamma_{f} v_{g} \frac{1}{1+\left(\left(v_{g} \tau\right) / b\right)^{2}}, \\
& \frac{\delta \varphi_{L}}{\partial z}(0,0)=-\Gamma_{f} \frac{1}{1+\left(\left(v_{g} \tau\right) / b\right)^{2}}+\Gamma_{f} .
\end{aligned}
$$

Fig. 1 shows the dependences of the phase and its derivatives in time and coordinate under the conditions $z=0 \quad$ and $\quad t=\left\{-L /\left(2 v_{g}\right) ; L /\left(2 v_{g}\right)\right\} . \quad$ For better clearness, the values on the ordinates axis here and below are given in arbitrary units with normalized scale.

Now, let us consider the phase delay at the output side of the medium. In this case, the distribution of increase of the wave vector length $\Gamma$ remains unchanged. However, a wave front that came out of the point $z$ at time $t$ will get to the point $\xi$ (on the pass from $z$ to the output border) at $t^{\prime \prime}=t+\frac{\xi-z}{v_{\mathrm{ph}}}=t-\frac{z-\xi}{v_{\mathrm{ph}}}$, where $v_{\text {ph }}$ is the phase velocity of scattered radiation.

Expressions for $t^{\prime}$ and $t^{\prime \prime}$ are identical and differ only by the values of speed, thus:

$$
\delta \varphi_{\omega}(t, z)=\Gamma_{f} \int_{z}^{L / 2} \exp \left[-(\xi / b)^{2}\right] \exp \left[-\left(\frac{t-(z-\xi) / \nu_{\mathrm{ph}}}{\tau}\right)^{2}\right] d \xi .
$$

As $L / 2>>b$, the upper limit of integral (10) can be replaced with $+\infty$. Let us calculate $\delta \varphi_{\omega}$ for conditions specified earlier $z=0, t=0$ :

$$
\begin{aligned}
& \delta \varphi_{\omega}(0,0)=\Gamma_{f} \int_{0}^{\infty} \exp \left[-(\xi / b)^{2}-\left(\xi /\left(\tau v_{\mathrm{ph}}\right)\right)^{2}\right] d \xi= \\
& =\frac{\sqrt{\pi}}{2} \Gamma_{f} b \frac{1}{\sqrt{1+\left(b /\left(\tau \mathrm{v}_{\mathrm{ph}}\right)\right)^{2}}} .
\end{aligned}
$$

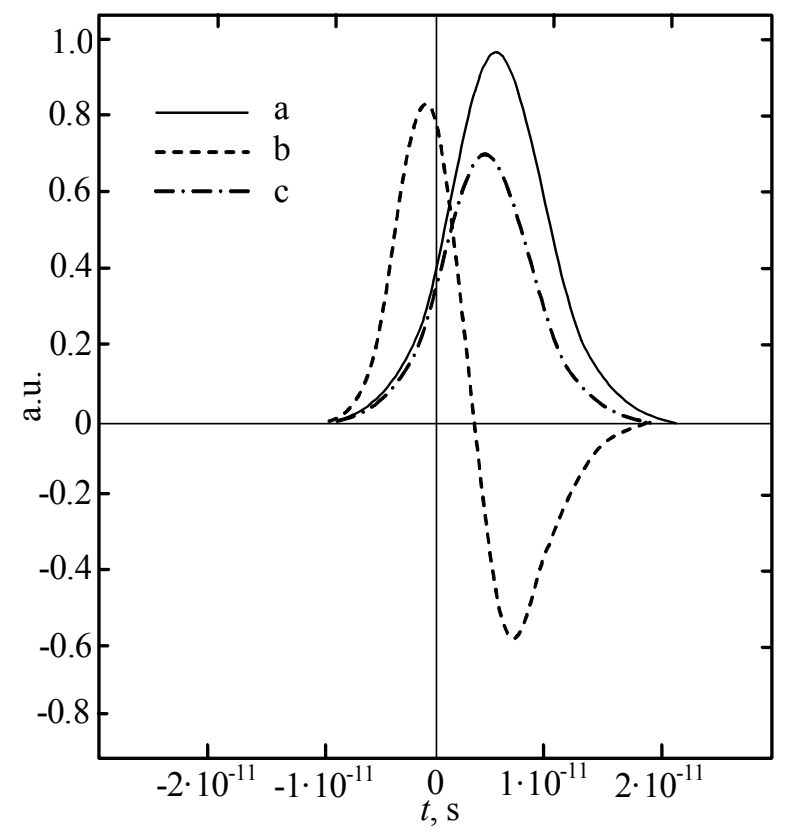

Fig. 1. Phase delay of the laser radiation at the input side of the medium (a) and its derivatives on time (b) and coordinate (c) in arbitrary units. 
Similarly, we calculate the derivatives on time and coordinate at the output side of the medium:

$$
\begin{aligned}
& \frac{\partial \varphi_{\omega}}{\partial t}(0,0)=-\Gamma_{f} \mathrm{v}_{\mathrm{ph}} \frac{1}{1+\left(\left(\mathrm{v}_{\mathrm{ph}} \tau\right) / b\right)^{2}}, \\
& \frac{\partial \varphi_{\omega}}{\partial z}(0,0)=\Gamma_{f} \frac{1}{1+\left(\left(\mathrm{v}_{\mathrm{ph}} \tau\right) / b\right)^{2}}-\Gamma_{f} .
\end{aligned}
$$

The phase and its derivatives, obtained using (10), are shown in Fig. 2 under the same conditions as for the input (laser) radiation.

The total phase delay and its derivatives could be found as a sum of specified values at the input and output sides of the medium:

$$
\delta \varphi(0,0)=\frac{\sqrt{\pi}}{2} \Gamma_{f} b\left(1 / \sqrt{1+\left(\frac{b}{\tau \mathrm{v}_{g}}\right)^{2}}+1 / \sqrt{1+\left(\frac{b}{\tau \mathrm{v}_{\mathrm{ph}}}\right)^{2}}\right),
$$

$$
\begin{aligned}
& \frac{\partial \delta \varphi}{\partial t}(0,0)=-\Gamma_{f} \frac{\mathrm{v}_{\mathrm{ph}}}{1+\left(\frac{\mathrm{v}_{\mathrm{ph}} \tau}{b}\right)^{2}}+\Gamma_{f} \frac{\mathrm{v}_{g}}{1+\left(\frac{\mathrm{v}_{g} \tau}{b}\right)^{2}}, \\
& \frac{\partial \delta \varphi}{\partial z}(0,0)=\Gamma_{f} \frac{1}{1+\left(\frac{\mathrm{v}_{\mathrm{ph}} \tau}{b}\right)^{2}}-\Gamma_{f} \frac{1}{1+\left(\frac{\mathrm{v}_{g} \tau}{b}\right)^{2}} .
\end{aligned}
$$

Resulting dependences are shown in Fig. 3 under the earlier accepted values $z$ and $t$.

Taking into account the above considerations, the analytical calculation of polarization phase can be simplified by using the results (14) and (15) for derivatives and linear interpolation:

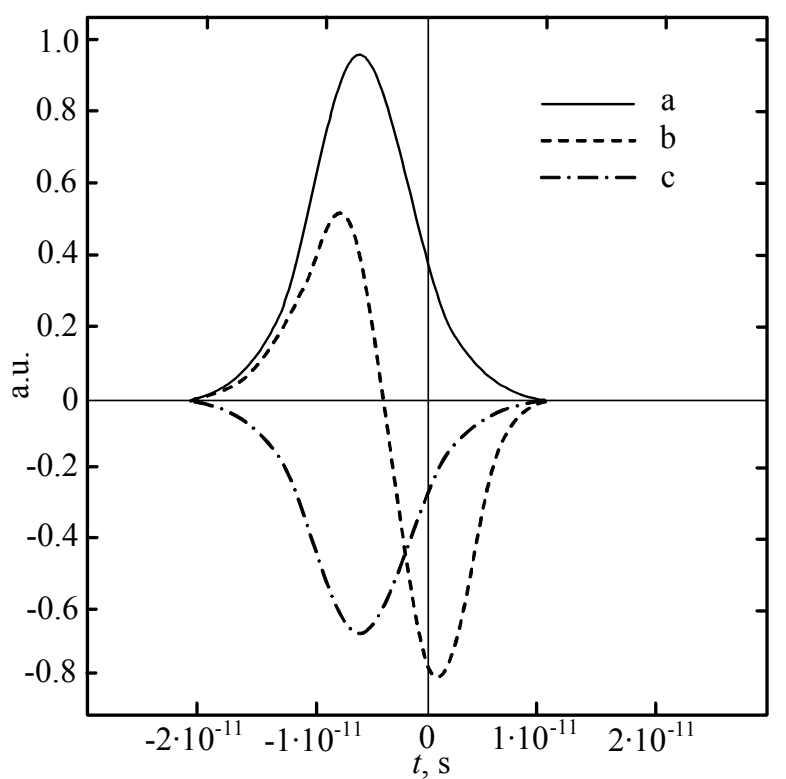

Fig. 2. The phase delay of the scattered radiation at the output side of the medium (a) and its derivatives on time (b) and coordinate (c) in arbitrary units.

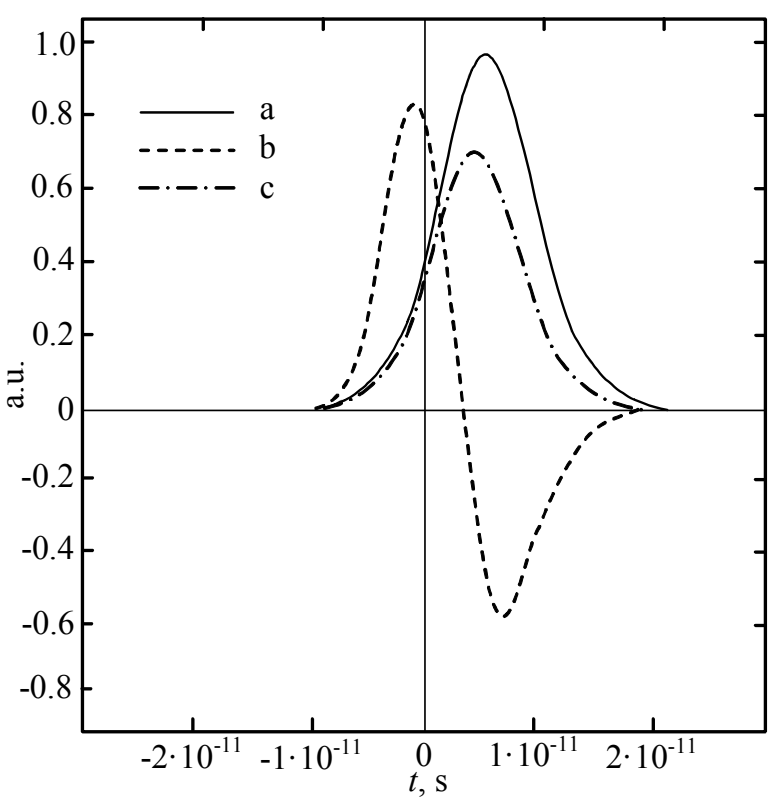

Fig. 3. The total phase delay of the scattered radiation (a) and its derivatives on time (b) and coordinate (c) in arbitrary units.

$$
\begin{aligned}
& \varphi_{p}=\omega_{0} t-k_{p} z-\delta \varphi_{L}-\delta \varphi_{\omega}= \\
& =\left(\omega_{0}-\Gamma_{f} \mathrm{v}_{g} \frac{1}{1+\left(\left(\mathrm{v}_{g} \tau\right) / b\right)^{2}}+\Gamma_{f} \mathrm{v}_{\mathrm{ph}} \frac{1}{1+\left(\left(\mathrm{v}_{\mathrm{ph}} \tau\right) / b\right)^{2}}\right) t- \\
& -\left(k_{p}-\Gamma_{f} \frac{1}{1+\left(\left(\mathrm{v}_{g} \tau\right) / b\right)^{2}}+\Gamma_{f} \frac{1}{1+\left(\left(\mathrm{v}_{\mathrm{ph}} \tau\right) / b\right)^{2}}\right) z,
\end{aligned}
$$

where $\varphi_{p}$ is the phase of polarization, $k_{p}$ - initial wave vector length of polarization without self-phase modulation and $\omega_{0}$ - initial frequency.

The expression (16) allows estimating the influence of self-phase modulation on the resulting wave vector length and frequency.

In case of toluene, the group velocity of ruby laser light is $v_{g}=0.65 c$, refractive index $n_{L}=1.49$, i.e. $v_{\mathrm{ph}}=$ $c / n_{L}=0.67 c$, relaxation time, as stated earlier, is $\tau=5 \mathrm{ps}$ and, finally, length of the focal area $b=0.1 \mathrm{~cm}$. Under these parameters the following values are obtained:

$$
\begin{aligned}
& \left(\frac{v_{g}}{1+\left(\left(v_{g} \tau\right) / b\right)^{2}}-\frac{v_{\mathrm{ph}}}{1+\left(\left(v_{\mathrm{ph}} \tau\right) / b\right)^{2}}\right) / 2 \pi c \approx 5 \cdot 10^{-6}, \\
& \frac{1}{1+\left(\left(\mathrm{v}_{g} \tau\right) / b\right)^{2}}-\frac{1}{1+\left(\left(\mathrm{v}_{\mathrm{ph}} \tau\right) / b\right)^{2}} \approx 0.01,
\end{aligned}
$$

meaning that in the first approximation the self-phase modulation of polarization can be neglected, i.e. (16) can be written as $\varphi_{p}=\omega_{0} t-k_{p} z$.

Functions $F_{t}(t), F_{\perp}\left(r_{\perp}\right), F_{z}\left(z-z_{f}\right)$ in (1) can be specified as Gaussian functions with according 
halfwidths $\tau, a_{f}, b$ at the level $e^{-1}$; also $z_{f}=0$ (the condition of stationary focal area).

Using (1), (5) and taking into consideration $\varphi_{p}=\omega_{0} t-k_{p} z$, we find that the frequency-angular density of radiation energy for parametric components is described by the expression:

$W_{\omega \theta}=\frac{\pi^{2} \omega^{4} n}{8 c^{3}}\left(P_{0}^{N L}\right)^{2} \tau^{2} a_{f}^{4} b^{2} \times$

$\times \exp \left[-0.5\left(\Delta k_{\perp}^{2} a_{f}^{2}+\Delta k_{\|}^{2} b^{2}+\Delta \omega^{2} \tau^{2}\right)\right]$,

where $\Delta k_{\|}=k(\omega) \cos \theta-k_{p}$ and $\Delta k_{\perp}=k(\omega) \sin \theta \quad$ are wave vector difference projections onto axis $z$ and axis perpendicular to it, i.e. the longitudinal and lateral mismatch of wave vector $\mathbf{k}(\omega)$ of the parametric components radiation and wave vector $\mathbf{k}_{p}$ of polarization; $\theta$ is the scattering angle determined by the angle between vector $\mathbf{k}(\omega)$ and axis $z$, along which the polarization wave vector is oriented.

The most optimal conditions to generate radiation are obtained, when the sum

$F=\Delta k_{\perp}^{2} a_{f}^{2}+\Delta k_{\|}^{2} b^{2}+\Delta \omega^{2} \tau^{2}$

in (6) gains its minimum value. The search for these conditions can be done through finding an extremum $d F / d \omega=0$. In the approximation of linear dispersion and small $\theta$, the energy density maximum $W_{\omega \theta}$ is shifted relative to the Raman frequency $\omega_{0}$ as

$\Delta \omega=\omega-\omega_{0}=-\Delta k_{0} \cup_{g} \frac{1-\frac{\theta^{2}}{\theta_{0}^{2}}\left(1+\Delta k_{0} / k_{0}-2 a_{f}^{2} / b^{2}\right)}{\theta^{2}\left(a_{f}^{2} / b^{2}-1\right)+1+\left(\tau v_{g} / b\right)^{2}}$,

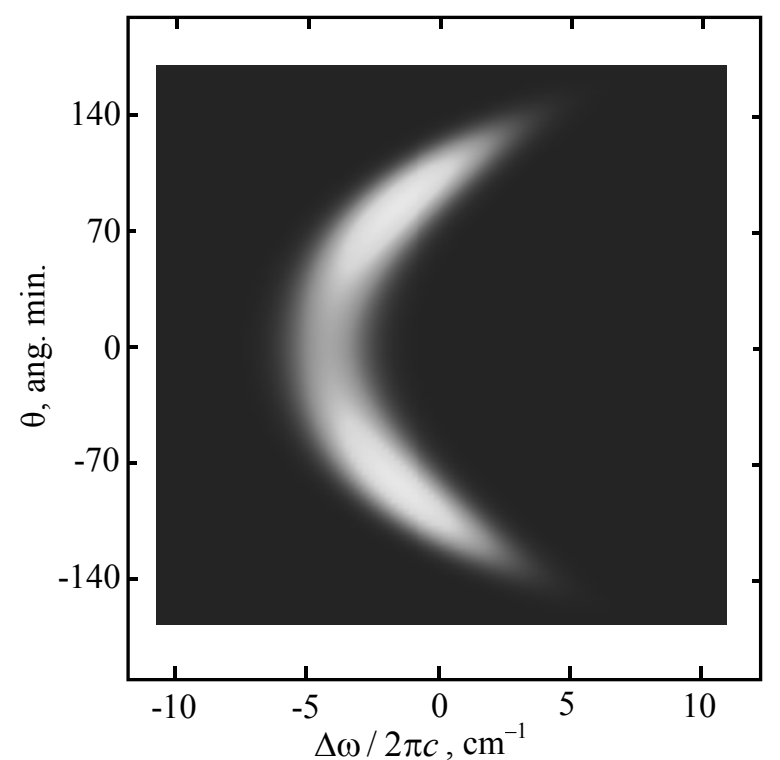

Fig. 4. Frequency-angular spectrum of the first anti-Stokes component in toluene.

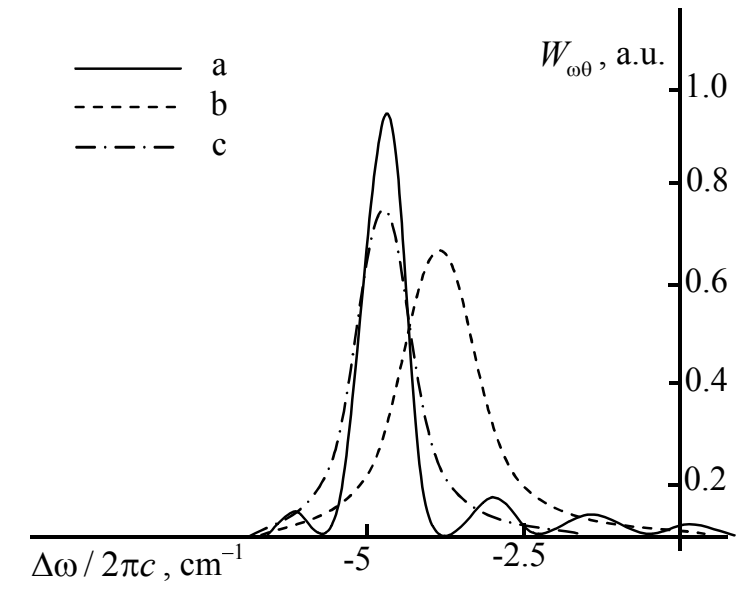

Fig. 5. Comparison of axial radiation energy density, when self-phase modulation is considered (a) and neglected: (b) for $b=0.1 \mathrm{~cm}$, (c) for $b=0.3 \mathrm{~cm}$.

where $\Delta k_{0}=k_{0}\left(\omega_{0}\right)-k_{p}$ is the difference of wave vector length of radiation $\left(k_{0}\right)$ at Raman frequency $\omega_{0}$ and wave vector length of polarization $\left(k_{p}\right) ; \theta_{0}=$ $\sqrt{2 \Delta k_{0} / k_{0}}$ is the scattering angle, for which $\Delta k_{\|}\left(\omega_{0}\right)=0$.

Additional simplification under conditions $a_{f}<<b, \Delta k_{0}<<k_{0}$ provides:

$\Delta \omega=-\Delta k_{0} \cup_{g} \frac{1-\left(\theta / \theta_{0}\right)^{2}}{1-\left(\tau v_{g} / b\right)^{2}}$.

Using (17) we can calculate a normalized spectrum of the first anti-Stokes component, when $k_{p}=2 k_{L}-k_{s}$, $\omega_{0}=2 \omega_{L}-\omega_{s}$, where $\omega_{s}$ and $k_{s}$ are the Stokes Raman frequency and wave vector length, $\omega_{L}$ and $k_{L}$ are laser radiation frequency and wave vector length, respectively. In toluene with SRS excitation by ruby laser, the following values are used: frequency of exciting radiation $\omega_{L} /(2 \pi c)=14403 \mathrm{~cm}^{-1}$; frequency of Raman-active vibration $\Omega=1004 \mathrm{~cm}^{-1}$; focal area length $b=0.1 \mathrm{~cm}$; focal area radius $a_{f}=5 \mu \mathrm{m}$; existence duration of nonlinear polarization $\tau=2.5 \mathrm{ps}$.

The resulting frequency-angular spectrum structure is shown in Fig. 4.

Finally, let us estimate in more detail, how selfphase modulation affects the spectrum characteristics, for example, of the axial radiation. The energy density that accounts self-phase modulation can be calculated using (5) and (16), which was done numerically. After that, the calculated spectrum that accounts self-phase modulation and the one obtained from (17) are compared under varying values of $b$, as one of the focal area 
characteristics. Comparison of the normalized spectra for axial radiation is shown in Fig. 5. As can be seen, the larger is $b$, the closer match results obtained between spectrum that accounts self-phase modulation and that neglecting it.

\section{Conclusions}

The obtained results indicate that self-phase modulation in the self-focusing and relaxation oscillations mode has a weak effect on the radiation spectra of SRS. Nevertheless, parametric components have the frequency-angular structure, shaped as parabolic $\omega-\theta$ branches. The shift of the parametric component peak energy in spectrum, obtained in media with normal dispersion at transparency area, is determined by the size of the focal area, its existence duration, and mismatch $\Delta k_{0}$ between polarization and wave vectors field of the corresponding parametric component at the frequency of exact Raman resonance.

The important point is that the generation of radiation at shifted frequencies occurs under short-term phase matching, and it is a non-stationary process. The principal difference between amplitude-phase matching [6] and short-term phase matching is that in the first case the area of the maximum polarization amplitude moves, and matching of the polarization phases and the field, which is generated at a shifted frequency, remains constant in the case of steady motion. In the case of short-term phase, matching the area of the polarization amplitude is stationary, and therefore matching of phases is rapidly disturbed.

Influence of the short-term phase matching conditions $(\Delta \mathbf{k}=0, \Delta \omega \neq 0)$ can cause a frequency shift of radiation in other non-stationary processes, not related to SRS. In particular, this relates to the frequency doubling of picosecond focused light pulses in samples, which length is much shorter than that of the pulse, in powdery nonlinear optical media, or on the surface of absorbing media by "slide reflection" scheme, etc.

\section{References}

1. V.I. Bespalov, G.A. Pasmanik, Nonlinear Optics and Adaptive Laser Systems. Moscow, Nauka, 1985 (in Russian).

2. G.S. Felinskiy, P.A. Korotkov, Simulation of multiwave pumped fiber Raman amplifiers // Semiconductor Physics, Quantum Electronics \& Optoelectronics, 9(3), p. 35-39 (2006).

3. C.A. Sacchi, C.H. Townes, J.R. Lifshitz, AntiStokes generation in trapped filaments of light // Phys. Rev. 174(2), p. 439-448 (1968).

4. N.E. Kornienko, V.I. Maliy, G.V. Ponezha, E.A. Ponezha, The nature of the frequency-angular structure of radiation in stimulated Raman scattering in liquids // Izvestiia Akademii Nauk UkrSSR, Ser. A, 4, p. 65-68 (1983), in Russian.

5. A.I. Ivanisik, V.I. Maliy, G.V. Ponezha, Cherenkov-type radiation under conditions of Raman light scattering in self-focusing liquids // Optics and Spectroscopy, 82(3), p. 410-416 (1997).

6. A.I. Ivanisik, V.I. Maliy, G.V. Ponezha, Spectrum of anti-Stokes stimulated Raman scattering from the moving focal regions of self-focusing // Optics and Spectroscopy, 90(4), p. 625-629 (2001).

7. Y.R. Shen, The Principles of Nonlinear Optics. New York, Wiley, 1984.

8. A.I. Ivanisik, V.I. Maliy, G.V. Ponezha, Effect of self-focusing on the angular spectra of stimulated Raman scattering // Optics and Spectroscopy 85(1), p. 78-84 (1998).

9. R.V. Johnson, J.H. Marburger, Relaxation oscillation in stimulated Raman and Brillouin scattering // Phys. Rev. A, 4(3), p. 1175-1182 (1971). 